\title{
ON THE IMPACT OF FIBER NONLINEAR EFFECTS ON THE CP-DQPSK MODULATED ULTRA-DWDM SYSTEM
}

\author{
Tomáš HUSZANÍK, Ján TURÁN, Luboš OVSENÍK \\ Department of Electronics and Multimedia Communications, Faculty of Electrical Engineering and Informatics, \\ Technical University of Košice, Němcovej 32, 04001 Košice, Slovak Republic, Tel.: +421 556024277 , \\ E-mail: tomas.huszanik@tuke.sk,jan.turan@tuke.sk, lubos.ovsenik@tuke.sk
}

\begin{abstract}
The exponential increase in transmission capacity triggered the era of fast and reliable data transfer techniques through fiber optic networks. This has led to the exploration of new options in the field of spectrally efficient systems suitable for extremely high data rates. Consequently, there is a gradual transition from existing systems with a 10 Gbps transmission rate to 40 Gbps optical systems and even up to 100 Gbps. However, with the increase in network transmission capacity, the optical signal performance requirement is also increased to ensure acceptable bit error rate (BER) in the receiver. However, increasing transmission power leads to a distortion of the transmitted signal due to the nonlinear Kerr effects or interference between adjacent channels. These are the main limitations that set the upper limit of the maximum effective SNR (Signal-to-Noise Ratio) of the optical link and limit the performance of the whole system. On the other hand, linear effects such as chromatic dispersion (CD), optical SNR (OSNR), spontaneous photon emission, optical fiber loss, or channel overlapping through long stretches of optical fibers are also important factors that need to be considered when designing DWDM (Dense Wavelength Division Multiplexing) system.
\end{abstract}

Keywords: advanced optical modulation, CP-DQPSK, DWDM, fiber nonlinear effects

\section{INTRODUCTION}

Despite relatively reliable and efficient modulation formats (intensity modulation), the spectral efficiency of the DWDM system is heavily influenced by nonlinear effects such as self-phase modulation (SPM), cross-phase modulation (XPM) and four-wave mixing (FWM). Nonlinear effects affect not only the backbone networks, but also access fiber optic networks and their capacity and transmission range itself. Dominant constraints in optical access networks are embedded damping in passages of the optical path with passive network components, linear overlaps in multiplexing and demultiplexing devices, but also attenuation in optical fibers [1-2].

To increase the spectral efficiency and energy efficiency of the DWDM system, a wide range of techniques has been proposed. One of these techniques is efficient bandwidth management where the performance of the DWDM system is controlled by increasing/decreasing transmission rate per channel or by increasing/decreasing the number of channels or inter-channel spacing. Another technique that can increase the performance of the DWDM system is the use of advanced modulation techniques that not only improve the signal to noise ratio, but are also more resistant to fiber nonlinear effects and chromatic dispersion. Increasing the spectral efficiency and energy efficiency of the DWDM system is also possible by optimizing the design of the system - accurate analytical modeling and simulation of the optical link, the use of advanced algorithms for editing DSP-based signals, signal reception equalization or Forward Error Coding (FEC). Energy efficiency can also be improved by minimizing the required average signal or OSNR at a given BER level. Signal efficiency is measured in bit/s/Hz and can be increased using different spectral-effective modulations, which is also the focus of this work. The main aim of this research work is to combine the above methods to increase the spectral and energy efficiency of the DWDM system by means of spectral-effective optical modulation [3-5].

With the new 40 Gbps, 100 Gbps and 400 Gbps DWDMs there is a need to increase spectral efficiency. Such way is through the advanced optical modulation. The optical DPSK (Differential Phase Shift Keying) modulation is widely used in 40 Gbps DWDM systems. This approach is a combination of mixed ASK (Amplitude Shift Keying) modulation and PSK (Phase Shift Keying) modulation.

DPSK brings the increased spectral efficiency over OOK (On-Off Keying), while still encoding 1 bit per symbol. DQPSK (Differential Quadrature Phase Shift Keying) encodes 4 bits per symbol. The digital coherent transmission enabled encoding more bits per symbol which reduces the spectral width of the signal. In the DWDM systems with the transmission rate beyond $100 \mathrm{Gbps}$, there is a need for even more capacity and spectral efficiency. Reasonable solution is a coherent polarization (CP) DQPSK (CP-DQPSK) which encodes 4 bits per symbol of each polarization tributary [3][4].

In this paper we analyse the impact of fiber nonlinear effects on a CP-DQPSK modulated Ultra-DWDM system. The performance of the proposed simulation model is mathematically analysed and BER values are calculated.

\section{FIBER NONLINEAR EFFECTS}

There are three major nonlinear effects that occur inside optical fiber - self-phase modulation (SPM), cross-phase modulation (XPM) and four-wave mixing (FWM). All these effects are governed by a nonlinear parameter $\gamma$ :

$$
\gamma\left(\omega_{0}\right)=\frac{\omega_{0} n_{2}}{c A_{\text {eff }}}
$$

where $\omega_{0}$ is the frequency at which the pulse frequency is centred, $n_{2}$ is the nonlinear-index coefficient, $c$ is the 
velocity of light in vacuum and $A_{\text {eff }}$ is the effective mode area. The effective mode area depends on the fiber design. Thus, with the proper design $\gamma$ can be enhanced. The nonlinear-index coefficient is fixed for each glass material and it is third-order susceptibility related.

Mentioned nonlinear effects - SPM, XPM, FWM arise due to the dependence of the refractive index of the optical fiber core on the intensity of the light beam propagating in the optical fiber [4]. This dependency is also called Kerr effect. The refractive index of the optical fiber core can be expressed as:

$$
n=n_{0}+n_{2} \cdot \frac{P}{A_{e f f}},
$$

where $n_{0}$ is the refractive index of the optical fiber core, $n_{2}$ is the nonlinear refractive index, $P$ is the power bound to the optical fiber. Kerr effect is manifested in various forms. On the basis of the shape and form of the input signal, it can be shown as SPM, XPM and FWM. The origin and the creation of fiber nonlinear effects is described by Singh in [4].

\subsection{SPM}

Self-phase modulation occurs through the dependence of the refractive index in a nonlinear optical medium of the light intensity bound to the optical medium. SPM is the phenomenon that leads to the spectral broadening of optical pulses. SPM describes frequency changes that are caused by changes in optical power in one optical communication line. The binary nature of the signal, which consists of the maximum and minimum power, occurs as so-called frequency chirp. This SPM-induced chirp affects the shape of the pulse, leading to its widening at the output. The intensity is highest at the peak of the impulse envelope, part of the pulse reaches the highest refractive index and consequently spreads more slowly than the front and end portions of the pulse. In general, this phenomenon is considered to be undesirable, but on the other hand we can use it for soliton transmissions (using specially shaped pulses that shape themselves by SPM).

The phase of the optical fiber propagated signal is expressed as:

$$
\phi=\frac{2 \pi}{\lambda} n_{1} L
$$

where $\lambda$ is the wavelength of the optical pulse, $n_{l}$ is the refractive index of the optical fiber core, and $L$ is the length of the optical fiber.

The intensity of the transmitted pulse is time dependent, then the pulse phase $\phi$ will be time dependent. Changing the pulse phase over time will cause a change in signal frequency:

$$
\Delta \varphi=-2 \pi n_{1} \frac{L}{\lambda_{0} A_{e f f}} P,
$$

where $n_{l}$ is the refractive index of the optical fiber core, $L$ is the optical fiber length, $A_{\text {eff }}$ is the optical fiber core area, and $P$ is the transmitted signal power. The phase shift of the signal due to SPM is dependent on the power bound to the optical fiber. The phase of the transmitted pulse affected by the SPM can then be expressed as follows: $\varphi(t)=\omega_{0}(t)-\frac{2 \pi}{\lambda_{0} n(I) L}$

where $\omega_{0}$ is the carrier optical frequency, $\lambda_{0}$ is the carrier wavelength, $n$ is the refractive index profile, and $L$ is the optical fiber length.

\subsection{XPM}

Dominant effects in multi-channel DWDM systems are XPM and FWM combined with chromatic and polarization dispersion. The effect of XPM is given by the ratio of power bound to the optical fiber $P$ and the effective surface of the optical fiber core $A_{\text {eff. }}$ Let's consider the N-channel DWDM system [5-7]. The phase shift of the i-th channel through the $\mathrm{XPM}$ effect is then as follows:

$$
\phi_{n l}^{i}=k_{n l} L_{e f f}\left(P_{i}+2 \sum_{n=i+1}^{N} P_{n}\right)
$$

where $k_{n l}$ is the nonlinear propagation coefficient, $L_{\text {eff }}$ is the effective length of the fiber and $P$ is the power. The first part of the equation (6) represents the contribution of the SPM and the second part the contribution of the XPM. The second part of equation (6) also expresses the nonlinear sensitivity, and indicates that XPM is two times more efficient at the same energy than the SPM [6-9].

\subsection{FWM}

FWM is the other dominant effect in multi-channel DWDM systems. The influence of the FWM is independent from the transmission rate. However, its influence is critically dependent on the channel spacing between adjacent channels and chromatic dispersion. FWM occurs at the propagation of at least three signals with different wavelengths. Due to the FWM, there is an interaction between the three distributed signals of the frequency $f_{i}, f_{j}$ and $f_{k}$, and a new frequency signal is generated [5][10]. The performance of the signal with respect to the length of the optical fiber affected by the FWM is:

$$
\begin{gathered}
P_{F}(L)=\frac{1024 \pi^{6}}{n^{4} \lambda^{2} c^{2}}\left(D_{X}\right) \cdot \frac{P_{i}(0) P_{j}(0) P_{k}(0)}{A_{e}^{2}} e^{-\alpha L} \\
\cdot \frac{\left(1-e^{-\alpha L}\right)^{2}}{\alpha^{2}} \eta
\end{gathered}
$$

In this relation $P_{i}, P_{j}$ and $P_{k}$ are the output powers of the optical fiber coupled signals, $P_{F}$ is the output of the newly generated filament frequency signal, $n$ is the optical fiber refractive index, $\lambda$ is the wavelength, $c$ is the velocity of light in the vacuum, $\alpha$ is the loss coefficient, $L$ is the length of the optical fiber, $D$ is the degradation factor, and $X$ is the nonlinear susceptibility.

Further description of nonlinear effect is in [4-11].

\section{SIMULATION MODEL AND RESULTS}

To analyze the impact of fiber nonlinear effects, CPDQPSK modulated Ultra-DWDM system was designed in OptiSystem $^{\mathrm{TM}}$. OptiSystem ${ }^{\mathrm{TM}}$ is the state-of-the-art simulation software used to design complex fiber communication systems. The simulation is based on 
variable bandwidth model. Signals propagate in the network as samples in a time domain with a user-selectable bandwidth. The variable bandwidth simulation (VBS) can be used with different fiber models: simplified model - takes into account only damping (fiber loss) or attenuation and dispersion (fiber linear effects). In both cases, the simulation is strictly linear; advanced model - VBS takes into account all fiber effects, both linear and nonlinear. This is the most comprehensive and effective simulation strategy, but it is more time consuming due to more complex mathematics [12].

First, the CP-DQPSK optical modulator was designed. The CP-DQPSK transmitter is based on 3 MZM (MachZehnder Modulator) DQSPK optical modulator shown in Fig. 1. The structure of CP-DQPSK transmitter is shown in Fig. 2. It is constructed of two optical DQPSK modulators based on $\mathrm{LiNbO}_{3}$ Mach-Zehnder modulators. Both DQPSK transmitters modulate two $100 \mathrm{Gbps}$ data stream (in I and Q plane). Each wavelength channel is transmitting on the two polarization states (combined by polarization combiner with $90^{\circ}$ polarization angle) and thus the resulting capacity of each wavelength channel is $400 \mathrm{Gbps}$ [12].

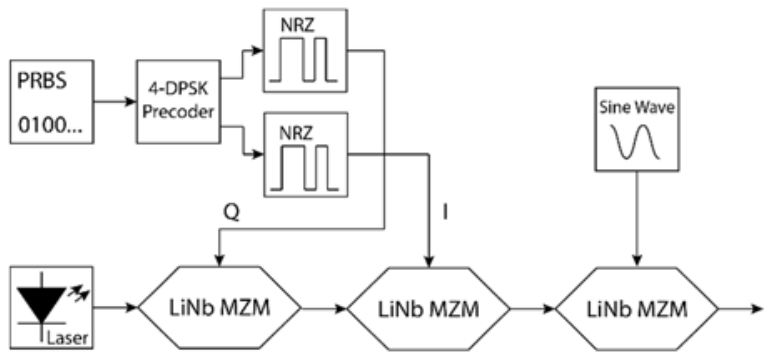

Fig. 13 MZM-based DQPSK modulator

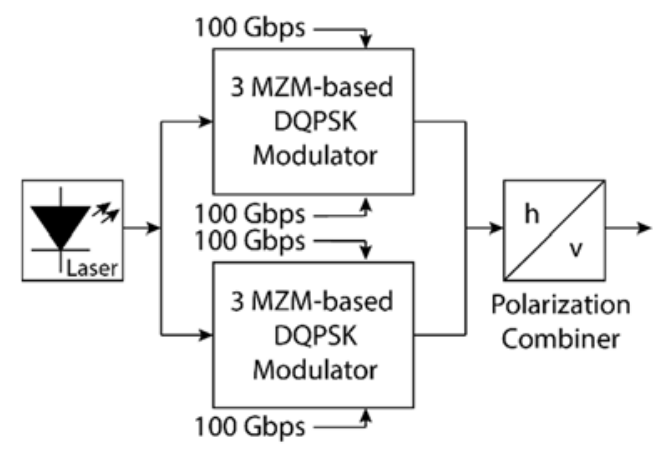

Fig. 2 CP-DQPSK transmitter

The block scheme of CP-DQPSK receiver/demodulator is in Fig. 3. Received signal is divided by polarization splitter with the polarization angle $90^{\circ}$, signals are then demodulated in a $90^{\circ}$ optical hybrid. Signal is finally converted to electrical signal by PIN photodiode. Received signal is analysed in electrical domain and from the received samples, the BER and Q-factor is calculated:

$$
Q=\frac{I_{1}-I_{0}}{\sigma_{1}+\sigma_{0}}
$$

In the equation (8), $I_{l}$ is the mean value and $\sigma_{l}$ is the deviation of the pulse $1, I_{0}$ is the mean value and $\sigma_{0}$ is the deviation of pulse 0 . The bit error rate (BER) is calculated as follows:

$$
B E R=\frac{1}{2} \operatorname{erfc}\left(\frac{Q}{\sqrt{2}}\right) \approx \frac{1}{\sqrt{2 \pi} Q} \exp \left(-\frac{Q^{2}}{2}\right) .
$$

The threshold for the BER value is $1.0 \mathrm{e}-10$ and for Qfactor 6.3613. The performance of proposed DWDM system is not acceptable for BER higher than 1.0e-10.

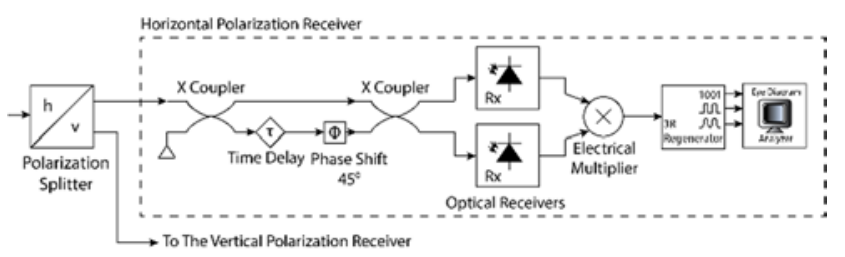

Fig. 3 CP-DQPSK receiver

\subsection{Effect of SPM on the signal propagation}

First, the impact of SPM on the signal propagation is analysed. In order to fully analyse the level of signal degradation caused by SPM we consider transmission through the highly nonlinear optical fiber (HNLF). The block scheme for modelling SPM is in Fig. 4. The source frequency is $193.1 \mathrm{THz}$. Bit rate is set to $100 \mathrm{Gbps}$. Chromatic dispersion is $16 \mathrm{ps} / \mathrm{nm} / \mathrm{km}$ and it is compensated using $10 \mathrm{~km}$ of dispersion compensating fiber (DCF).

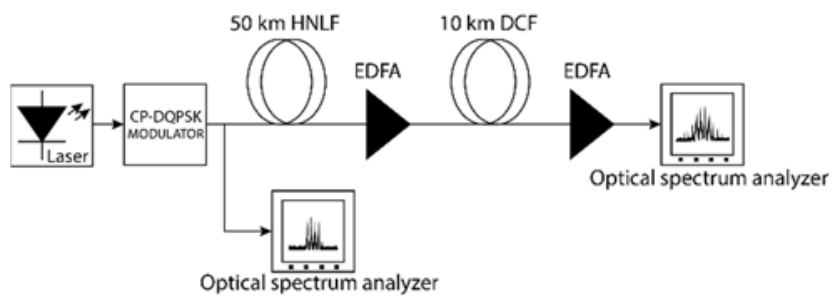

Fig. 4 Simulation model for modelling SPM

SPM is highly power dependent. It means that the change of optical power bound to the optical fiber result in a slight frequency change of transmitted pulse. This SPM-induced chirp affects the shape of the pulse, leading to its widening at the output. Fig. 5 shows the change of the pulse shape transmitted over $50 \mathrm{~km}$ of HNLF with the laser power set to $6 \mathrm{dBm}$ and $10 \mathrm{dBm}$.

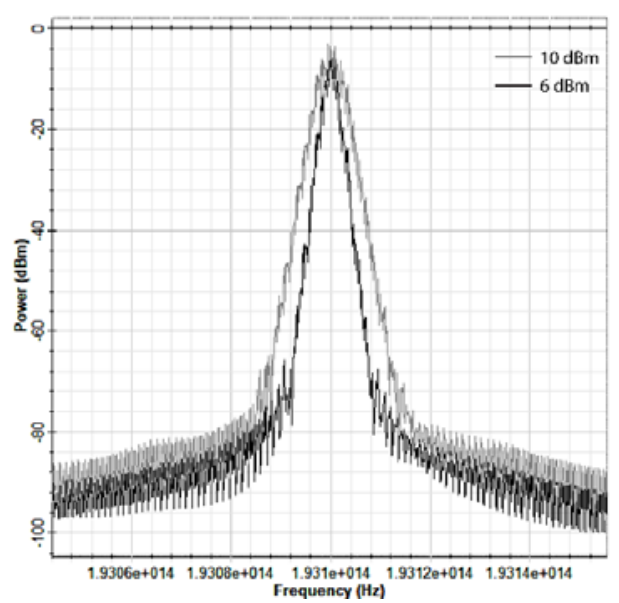

Fig. 5 SPM - change of pulse spectra for $6 \mathrm{dBm}$ and 10 $\mathrm{dBm}$ launch power 
The second simulation is designed to change transmission length by introducing another $50 \mathrm{~km}$ of HNLF and EDFA (Erbium Doped Fiber Amplifier) in the optical loop. By introducing another segment of HNLF and EDFA SPM is induced. The signal level remains the same, however, the spectra widens over longer distance. In multichannel transmission, this widening may cause serious damage, especially with tighter channel spacing. Dependence of SPM on the transmission distance is shown in Fig. 6.

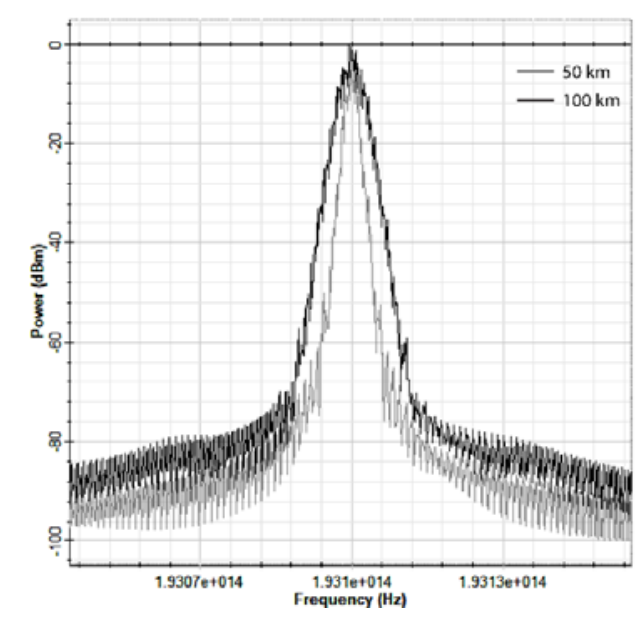

Fig. 6 SPM - change of transmission distance

\subsection{Effect of XPM and FWM on the signal propagation}

The simulation model for modelling XPM and FWM (shown in Fig. 7) is based on the simulation model shown in Fig. 4. In this case, two channel transmission takes place. Channel frequencies are $193.0 \mathrm{THz}$ and $193.1 \mathrm{THz}$, bitrate is set to $100 \mathrm{Gbps}$.

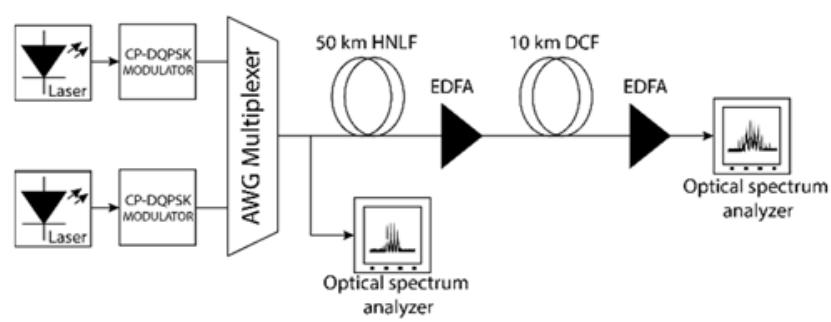

Fig. 7 Simulation model for modelling XPM and FWM

In the case of XPM and FWM the channel spacing is crucial. Channel spacing of DWDM system is defined by ITU-T G.694.1. Following figures (Fig. 8, Fig. 9, Fig. 10) show the creation of the new spectral components caused by combined effect of XPM and FWM using $25 \mathrm{GHz}$, $50 \mathrm{GHz}$ and $100 \mathrm{GHz}$ channel spacing between adjacent channels. It is obvious that the level of the newly generated spectral components due to the FWM depend on the channel spacing.

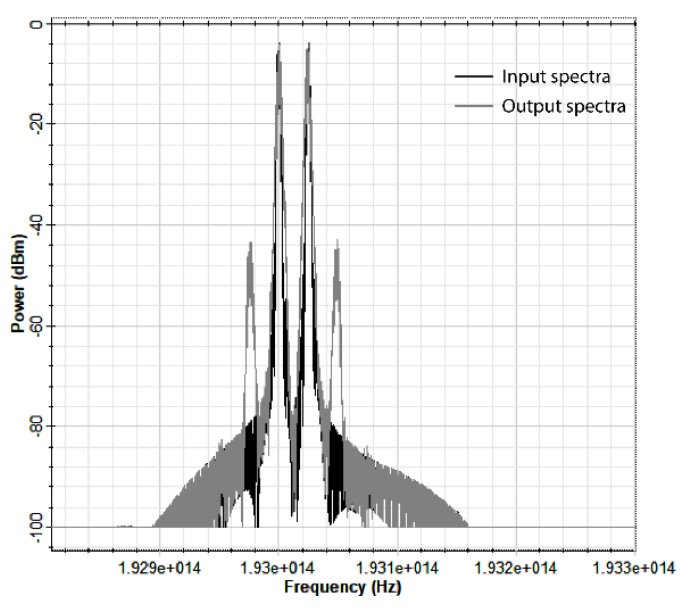

Fig. 8 Optical spectra using $25 \mathrm{GHz}$ channel spacing

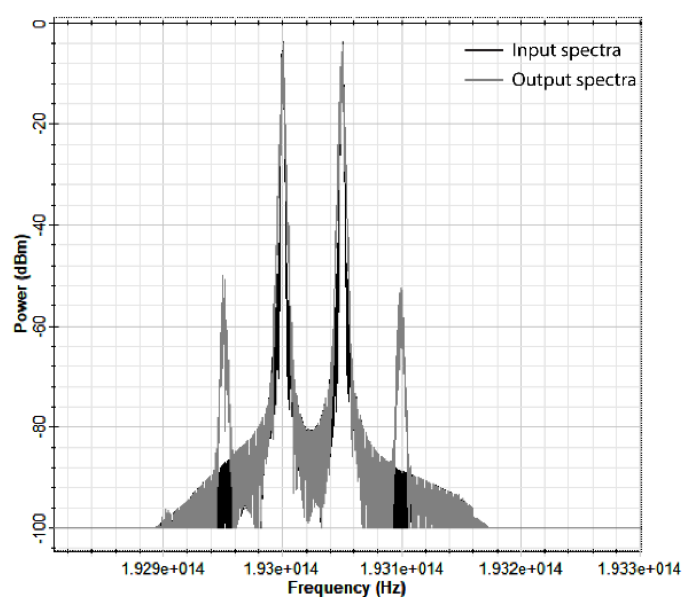

Fig. 9 Optical spectra using $50 \mathrm{GHz}$ channel spacing

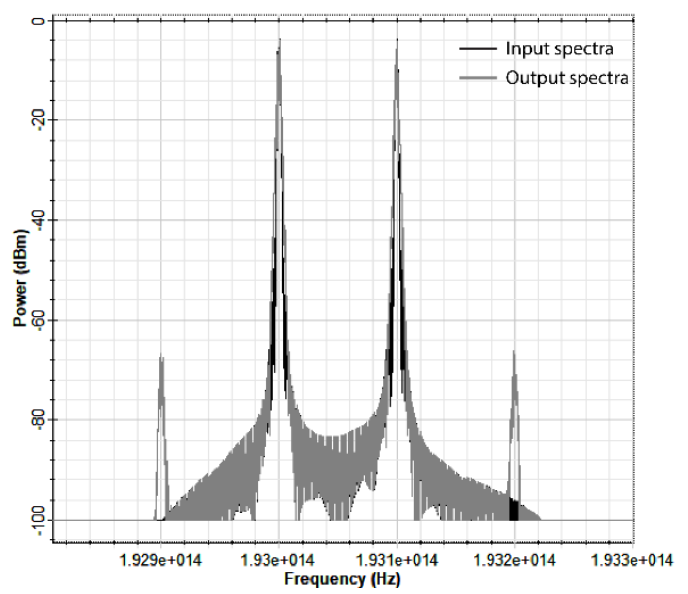

Fig. 10 Optical spectra using $100 \mathrm{GHz}$ channel spacing

As mentioned, XPM and FWM are dominant effects in multi-channel DWDM systems combined with chromatic and polarization dispersion. Fig. 11 shows the change in optical spectra depending on the value of chromatic dispersion. For chromatic dispersion of $0 \mathrm{ps} / \mathrm{nm} / \mathrm{km}$ the contribution of nonlinear effects increase. Optimal value of chromatic dispersion for proposed DWDM system is $16 \mathrm{ps} / \mathrm{nm} / \mathrm{km}$. 


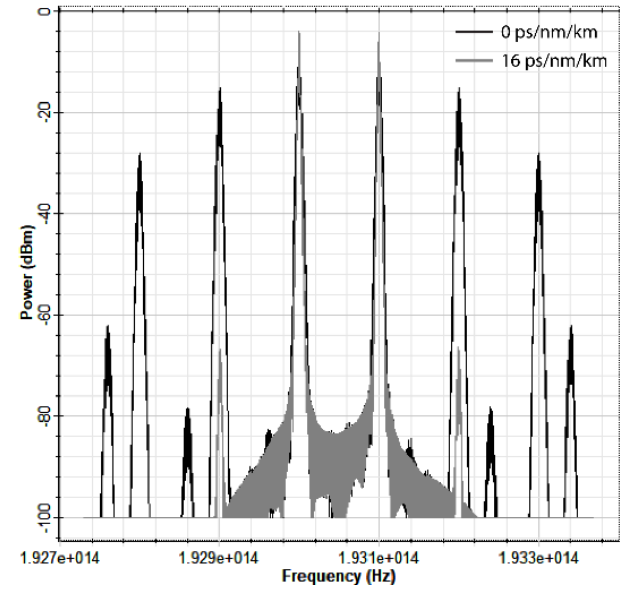

Fig. 11 The change of the optical spectra due to the chromatic dispersion

Finally, the change of the launch power of the two channels is evaluated. Similarly, as SPM, the increase of the launch power causes the widening of the optical spectra as seen in Fig. 12.

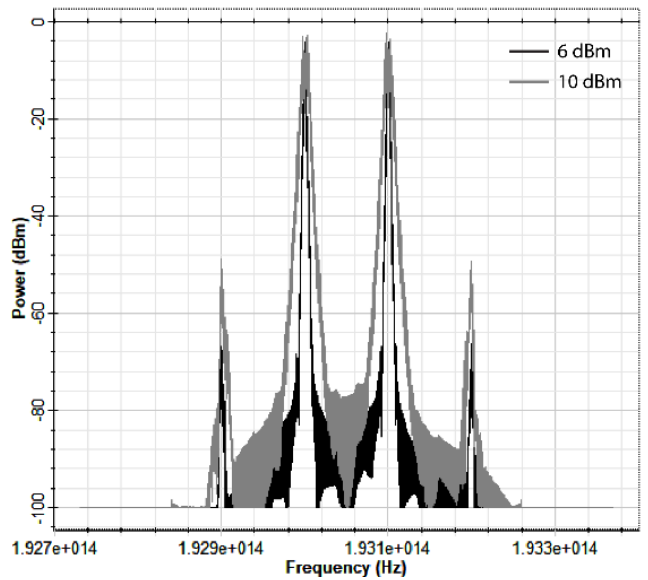

Fig. 12 The change of the optical spectra due to the laser launch power

\subsection{Evaluation of 32-channel Ultra-DWDM}

The simulation model of 32-channel Ultra-DWDM system is designed in OptiSystem ${ }^{\mathrm{TM}}$ simulation software.

The block scheme of the proposed simulation model is in Fig. 13. The simulation model consists of three subsections: transmitter section, optical distribution section and receiver section. The general simulation properties are: $100 \mathrm{Gbps}$ bit rate, time window is $2.56 \mathrm{e}-09 \mathrm{~s}$, sample rate $320 \mathrm{GHz}$ and sequence length $256 \mathrm{bit}$. The number of wavelength channels is 32 . The channel wavelengths are set according to the ITU-T G.694.1. The channel spacing is different throughout the various simulation setups: 200
$\mathrm{GHz}, 100 \mathrm{GHz}$ and $50 \mathrm{GHz}$. The simulation is done in an optical C band $(1530-1565 \mathrm{~nm})$. To increase the capacity of the whole DWDM system, optical CP-DQPSK is used at each wavelength channel. Channels are then multiplexed by 32-channel arrayed waveguide grating (AWG) multiplexer with the attenuation of $5.5 \mathrm{~dB}$.

Optical distribution path consists of $50 \mathrm{~km}$ of highly nonlinear single mode optical fiber (HNLF), two erbium doped fiber amplifiers (EDFA) and $10 \mathrm{~km}$ of dispersion compensation fiber (DCF). The optical distribution path is placed in the loop component. The number of loops is 20 $(1000 \mathrm{~km})$. The advanced simulation model of SMF is used. It means, that both linear and nonlinear effect are taken into account. The attenuation is $0.25 \mathrm{~dB} / \mathrm{km}$, chromatic dispersion is $16 \mathrm{ps} / \mathrm{km}-\mathrm{nm}^{2}$, nonlinear refractive index is $\mathrm{n}_{2}=2.6 \mathrm{e}-20 \mathrm{~m}^{2} / \mathrm{W}$ and effective cross section area of the fiber is $A_{\text {eff }}=80 \mu \mathrm{m}^{2}$. Chromatic dispersion is fully compensated in DCF with dispersion set to $-80 \mathrm{ps} / \mathrm{km}-\mathrm{nm}^{2}$. Channels are demultiplexed by AWG demultiplexer with $5.5 \mathrm{~dB}$ insertion loss. The maximum transmission distance is set to $1000 \mathrm{~km}$. Optical spectra of multiplexed signal at the beginning of the transmission path for $100 \mathrm{GHz}$ channel spacing is displayed in Fig. 14. Eye diagrams of received channel 5 are observed for three channel spacings: $50 \mathrm{GHz}$, $100 \mathrm{GHz}$ and $200 \mathrm{GHz}$. The creation of FWM is the most obvious in the $50 \mathrm{GHz}$ spaced DWDM system. The way to mitigate the influence of FWM is to increase the channel spacing. However, the effect of XPM is more dominant in this case. The XPM effect depends on the channels power and channel spacing. With in-line EDFA amplifying the XPM is induced. XPM has the strong phase influence on the adjacent channels. This phase mismatch is crucial for the successful detection and demodulation of CP-DQPSK signal.

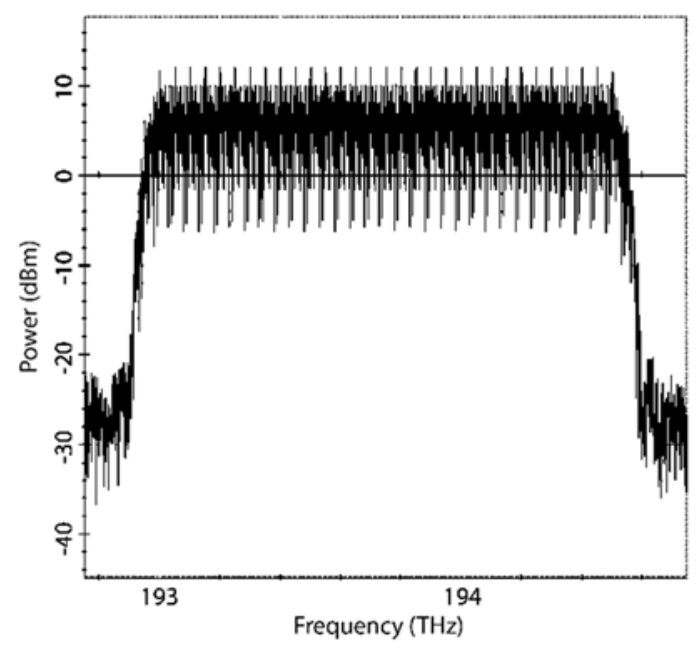

Fig. 14 Optical spectrums of transmitted signal 


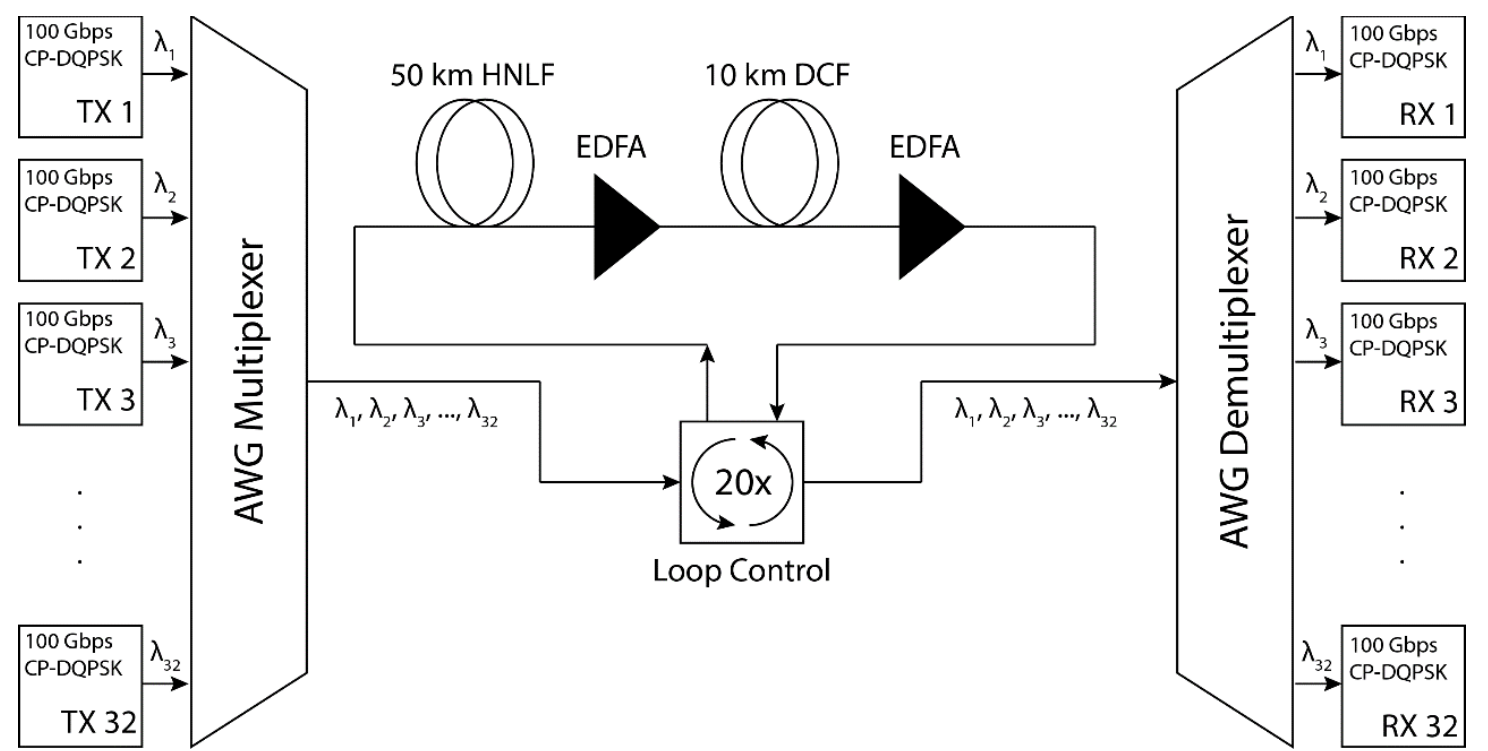

Fig. 13 Simulation model of 32-channel Ultra-DWDM system

The comparison of eye diagrams of $50 \mathrm{GHz}$ and $100 \mathrm{GHz}$ spaced DWDM are in Fig. 15. Lines in $50 \mathrm{GHz}$ eye diagram are thicker compared to the $100 \mathrm{GHz}$ eye diagram. This indicates the higher SNR (Signal-to-Noise Ratio) in the current sample point for the given channel. It is mainly caused by in-line amplifying and induced XPM effect. The BER value calculated from eye diagrams shown in Fig. 15 are: $1.89 \mathrm{e}-10(50 \mathrm{GHz})$ and 3.58e-15 (100 GHz).

Fig. 17 shows the dependence of different channel spacings on BER value. The difference in the performance between $\mathrm{H}$ and $\mathrm{V}$ polarization state of each simulation setup is very small. It indicates that the influence of the polarization mode dispersion (PMD) is small. The $200 \mathrm{GHz}$ spacing possesses the best performance, followed by $100 \mathrm{GHz}$ and $50 \mathrm{GHz}$ spacing. The maximum reach of 200 GHz spaced system is $800 \mathrm{~km}$ with BER: 5.51e-11 (H pol.) and $6.37 \mathrm{e}-10$ (V pol.). With the $100 \mathrm{GHz}$ channels spacing we can reach $640 \mathrm{~km}$ and BER: 1.82e-11 (H pol.) and 6.05e-10 (V pol.). The maximum reach of $50 \mathrm{GHz}$ spaced system is $560 \mathrm{~km}$ with BER: $1.89 \mathrm{e}-10(\mathrm{H} \mathrm{pol}$.) and $1.00 \mathrm{e}-$ 10 (V pol.).
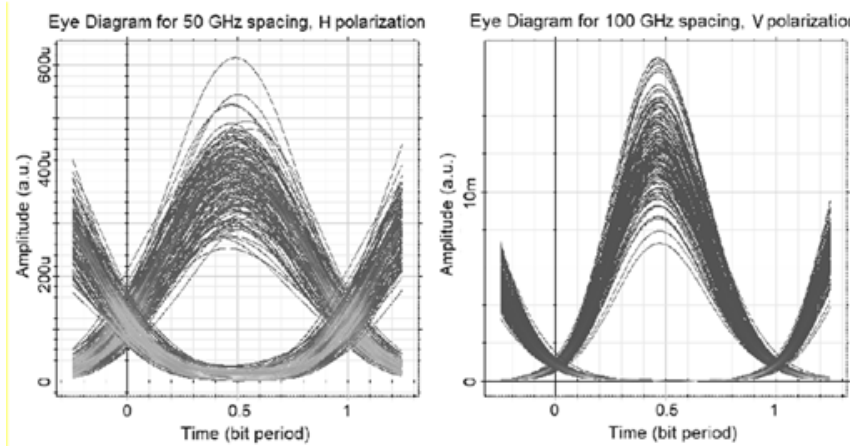

Fig. 15 Eye diagrams of received signals over $550 \mathrm{~km}(11$ loops) of optical fiber
In the next experiment, the effect of increased CW laser launch power on the creation of fiber nonlinear effects is analysed. The transmission distance is set to $600 \mathrm{~km}$ (12 loops). The CW laser launch power is linearly swept from $0 \mathrm{dBm}$ to $10 \mathrm{dBm}$. Again, the channel 5 is analysed and BER is calculated. Fig. 16 shows the eye diagrams of received channel 5 - for $100 \mathrm{GHz}$ and $50 \mathrm{GHz}$ channel spacing and $6.66 \mathrm{dBm} \mathrm{CW}$ laser launch power.
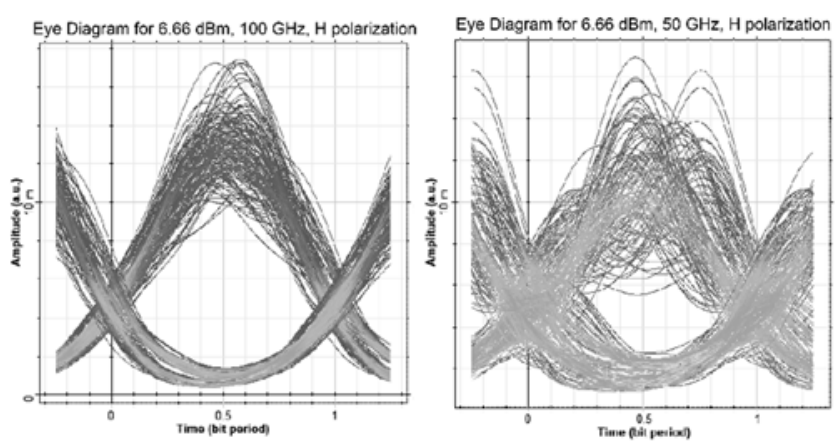

Fig. 16 Eye diagrams of received signals for $6.66 \mathrm{dBm}$ launch power

BER values calculated from eye diagrams shown in Fig. 17 are: $8.27 \mathrm{e}-009$ for $100 \mathrm{GHz}$ channel spacing and $1.08 \mathrm{e}-007$ for $50 \mathrm{GHz}$ channel spacing.

Fig. 18 shows the dependence of different launch level on the signal transmission over proposed CP-DQPSK modulated Ultra-DWDM system. Graphical dependence for both polarization states is provided. The difference of the performance between $\mathrm{H}$ and $\mathrm{V}$ polarization state is small. Thus it indicates the small contribution of PMD. The usable launch level of the proposed system is between 2 and $5 \mathrm{dBm}$ for $50 \mathrm{GHz}$ spaced DWDM, 1-6 dBm for $100 \mathrm{GHz}$ and $200 \mathrm{GHz}$ spaced DWDM. 

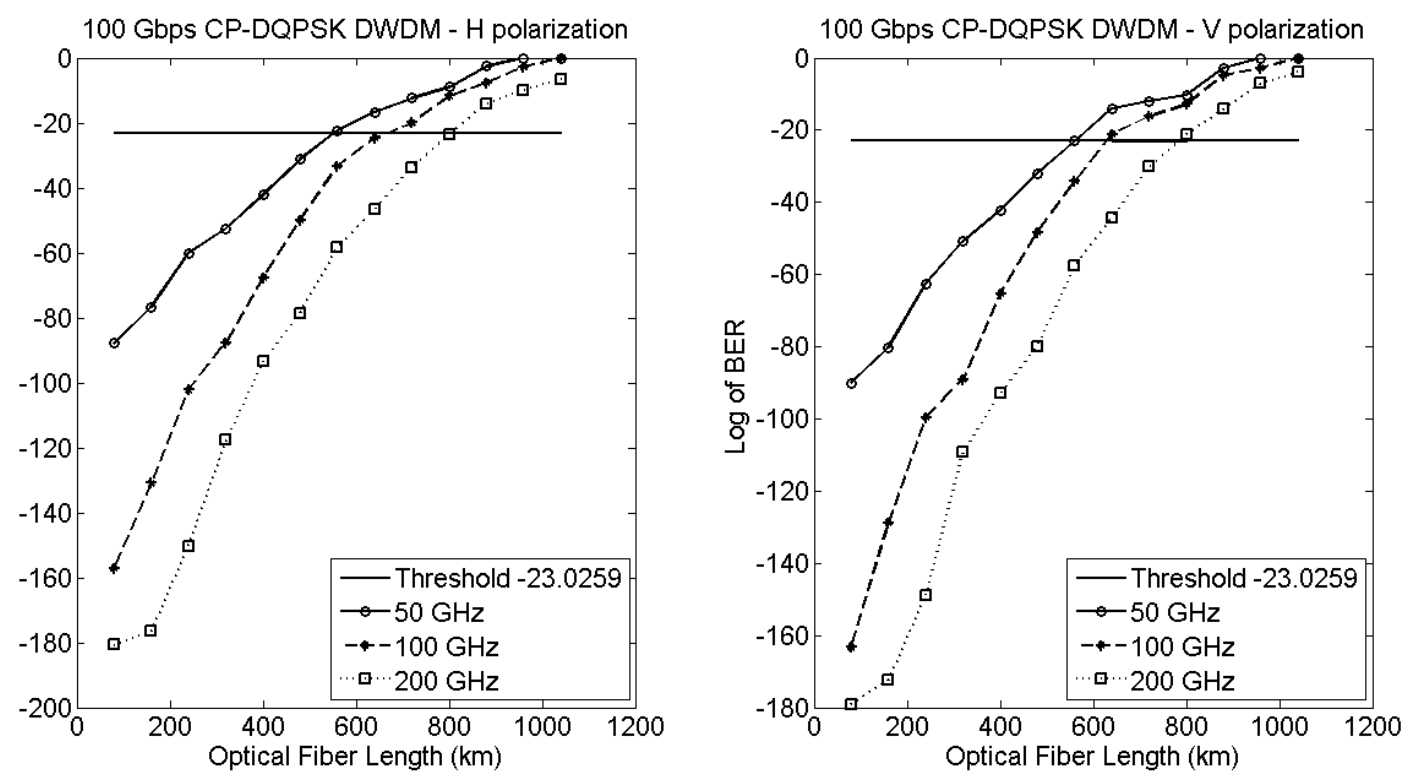

Fig. 17100 Gbps CP-DQPSK DWDM - BER vs different channel spacings
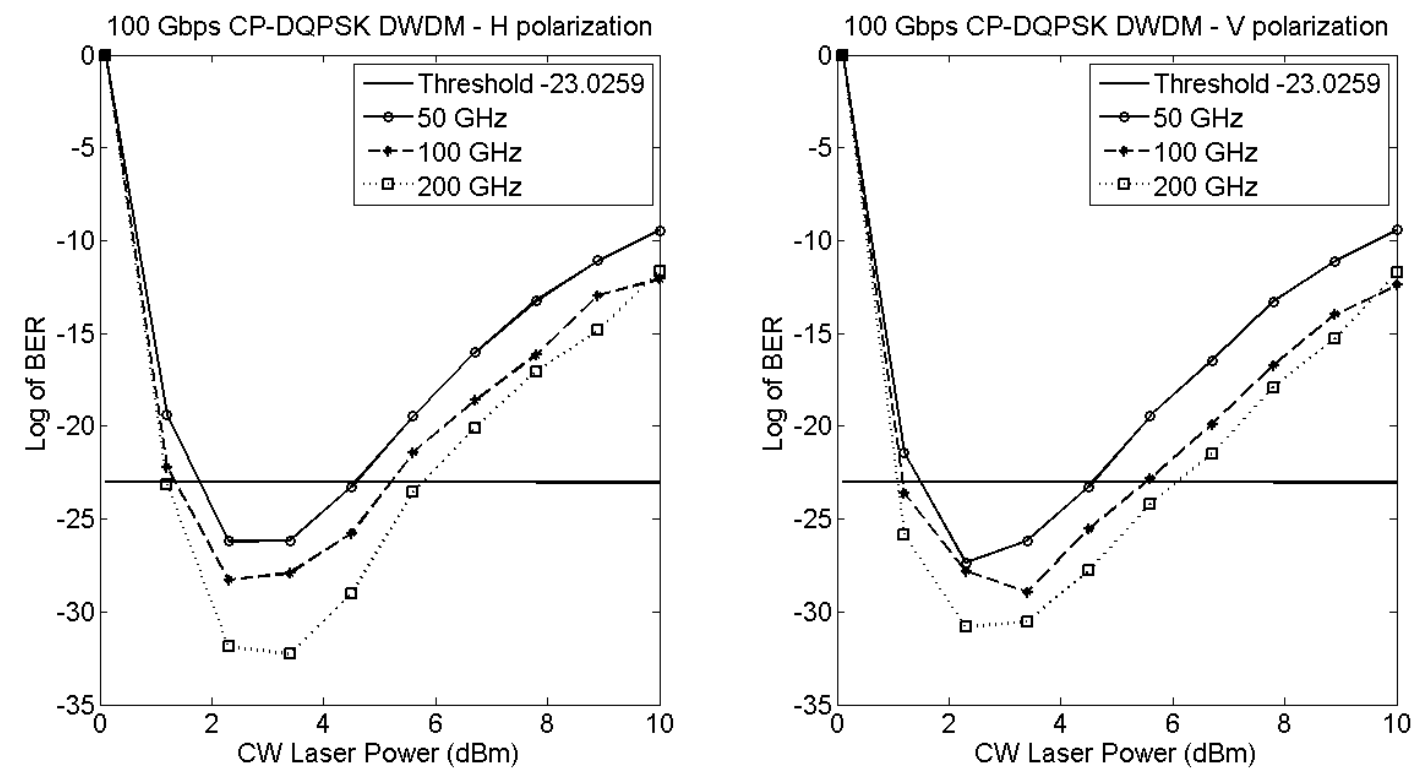

Fig. 18100 Gbps CP-DQPSK DWDM - BER vs CW launch power

\section{CONCLUSIONS}

The 32-channel Ultra-DWDM system with CP-DQPSK modulated signal and $400 \mathrm{Gbps}$ transmission rate per channel is analysed. In the simulation process we used the advanced mathematical model of optical fiber which enabled us to include nonlinear effects to the simulation model. We demonstrate that it is possible to use $50 \mathrm{GHz}$ channel spacing in such high capacity Ultra-DWDM system. The disadvantage is, of course, the lower achievable distance, in this case $500 \mathrm{~km}$. CP-DQPSK modulated DWDM system performs better with $200 \mathrm{GHz}$ and $100 \mathrm{GHz}$ compared to $50 \mathrm{GHz}$ channel spacing. Chromatic dispersion limits the transmission capacity when bit rate increases. It can be effectively compensated with DCF to extend the reach and improve the quality of the fiber optical line. The influence of fiber nonlinear effects is inevitable in long-haul transmissions when in-line optical amplifying is applied. To effectively transmit large number of bits we have not only to deploy advanced optical modulation technique such as CP-DQPSK, but also optimize the transmission path in term of reducing the effect of chromatic dispersion and fiber nonlinear effects.

\section{ACKNOWLEDGMENTS}

This work was supported by following research grants: KEGA 023TUKE-4/2017 and the Slovak Research and 
Development Agency under the contract no. "APVV-170208 - Resilient mobile networks for content delivery".

\section{REFERENCES}

[1] SIMMONS, J. M.: Optical Network Design and Planning, 2014, 529 p. ISSN: 1935-3847, DOI: $10.1007 / 978-3-319-05227-4$.

[2] KAZOVSKY, L. G. - SHAW, W. T. - GUTIERREZ, D. - CHENG, N. - WONG, S. W.: Next-Generation Optical Access Networks, Journal of Lightwave Technology, vol. 25, no. 11, 2017. DOI: 10.1109/JLT.2007.907748.

[3] KAUR, A. - DEWRA, S.: Comparative Analysis of Different Modulation Techniques in Coherent Optical Communication System, International Journal of Innovative Research in Computer and Communication Engineering, vol. 3, issue 8, pp. 7193 - 7200, 2015. ISSN: 2320-9801.

[4] SINGH, S. P. - SINGH, N.: Nonlinear Effects in Optical Fibers: Origin, Management and Applications, Progress In Electromagnetics Research, vol. 73, pp. 249-275, 2007.

DOI:10.2528/PIER07040201.

[5] MATSUMOTO, M. - OBATA, R.: Mitigation of cross-phase modulation in WDM transmission by mid-link electro-optic phase conjugation, 2017 OptoElectronics and Communications Conference (OECC) and Photonics Global Conference (PGC), Singapore, 2017, pp. 1-2.

DOI: 10.1109/OECC.2017.8114911.

[6] VIJAYAN, K. - FOO, B. - ELIASSON, H. ANDREKSON, P. A.: Cross-Phase Modulation Mitigation in WDM Transmission Systems Using Phase-Sensitive Amplifiers, 2018 European Conference on Optical Communication (ECOC), Rome, 2018, pp. 1-3.

DOI: $10.1109 /$ ECOC.2018.8535265.

[7] ARBOLEDA-ALZATE, N. - AMAYAFERNANDEZ, F.: Dispersion and nonlinear effects analysis for intensity and phase modulated optical signals, 2013 IEEE Colombian Conference on Communications and Computing (COLCOM), Medellin, 2013, pp. 1-6. DOI: 10.1109/ColComCon.2013.6564820.

[8] IVANIGA, T. - TURÁN, J. - OVSENÍK, L': Verification of the SPM Impact in DWDM System Using AWG Multiplexer / Demultiplexer, Acta Electrotechnica et Informatica, vol. 17, no. 1, 2017, pp. 17-22, DOI:10.15546/aeei-2017-0003.

[9] NAIN, H. - JADON, U. - MISHRA, V.: Performance investigation of Kerr effects on to WDM fiber optical networks, IEEE International Conference on Recent Trends in Electronics, Information and Communication Technology, 2016, pp. 2018-2022, DOI: 10.1109/RTEICT.2016.7808193.
[10] CHANG, J. - LIU, B. - ZHANG, L. - XIN, X. ZHANG, Q. - TIAN, Q. - YIN, X. - RAO, L. WANG, Y.: Polarization Mode Dispersion monitoring for phase-modulated optical signals utilizing parallel cross-phase modulation in a highly nonlinear fiber, 2016 15th International Conference on Optical Communications and Networks (ICOCN), Hangzhou, 2016, pp. 1-3. DOI: 10.1109/ICOCN.2016.7875715.

[11] HUSZANÍK, T. - TURÁN, J. - OVSENÍK, L.: Utilization of 10 Gbps DWDM System with Duobinary Modulation into Passive Optical Network, Journal of Communications Software and Systems, vol. 14, no. 4, 2018, pp. 367 - 385. DOI: http://dx.doi.org/10.24138/jcomss.v14i4.644.

[12] MOHAPATRA, S. K. - CHOUDHURY, R. R. BHOJRAY, R. - DAS, P.: Performance analysis and monitoring of various advanced digital modulation and multiplexing techniques of F.O.C within and beyond $400 \mathrm{~GB} / \mathrm{s}$, International Journal of Computer Networks \& Communications (IJCNC), vol.6, no.2, p. 159 - 181, India, 2014. ISSN: 0974-9322.

Received September 04, 2019, accepted November 14, 2019

\section{BIOGRAPHIES}

Tomáš Huszaník (Ing.) received Ing. (MSc.) degree with honors from multimedia communication in 2017 at Department of Electronics and Multimedia Communications, Faculty of Electrical Engineering and Informatics of Technical University of Košice. Since September 2017 he has been at Technical University of Košice as $\mathrm{PhD}$. student. His research interest is mainly focused on all optical fiber networks and mitigation and degradation mechanisms in all optical WDM systems.

Ján Turán (Dr.h.c., prof., RNDr., Ing., DrSc.) received Ing. (MSc.) degree in physical engineering with honours from the Czech Technical University, Prague, Czech Republic, in 1974, and RNDr. (MSc.) degree in experimental physics with honours from Charles University, Prague, Czech Republic, in 1980. He received a CSc. (PhD.) and DrSc. degrees in radioelectronics from University of Technology, Košice, Slovak Republic, in 1983, and 1992, respectively. Since March 1979, he has been at the Technical University of Košice, Slovak Republic as Professor for electronics and information technology. His research interests include digital signal processing and fiber optics, communication and sensing.

Luboš Ovseník (doc., Ing., PhD.) received Ing. (MSc.) degree in radioelectronics from the Technical University of Košice, in 1990. He received $\mathrm{PhD}$. degree in electronics from Technical University of Košice, Slovak Republic, in 2002. Since February 1997, he has been at the Technical University of Košice as Associate Professor for electronics and information technology. His general research interests include optoelectronic, photonics, fiber optic communications and fiber optic sensors. 